\title{
Tentative identification of formaldehyde in the Martian atmosphere
}

\author{
O. I. Korablev, 'M. Ackerman, ${ }^{2}$ V. A. Krasnopolsky, ${ }^{\prime}$ V. I. Moroz, ${ }^{\prime}$ C. Muller, ${ }^{2}$ A. V. Rodin ${ }^{1}$ and S. K. Atreya ${ }^{3}$ \\ Space Research Institute, Russian Academy of Sciences, Profsoyusnaya 84/32, 117810 Moscow GSP-7, Russia \\ Belgian Institute for Space Acronomy. Brussels. Belgium \\ ${ }^{3}$ Department of Atmospheric, Ocean and Space Sciences. University of Michigan. Ann Arbor, MI 48109-2143. U.S.A.
}

Recived 10 December 1992: revised 13 April 1993: accepted 13 May 1993

\begin{abstract}
Solar occultation observations of the Martian atmosphere near the limb of the planet were performed during the Phobos mission by means of the Auguste infrared spectrometer in the ranges $2707-2740$ and $5392-5272 \mathrm{~cm}^{-1}$ with a resolving power of $\approx 1300$. The spectra exhibit features at 2710 and $2730 \mathrm{~cm}^{-1}$ which have not been identified previously. After applying a set of corrections to the data and examining the spectra of various molecules, we are led to conclude that the best candidate for the above-mentioned features is formaldehyde $\left(\mathrm{CH}_{2} \mathrm{O}\right)$. It was observed in eight of the nine successful occultation sequences, mainly between 8 and $20 \mathrm{~km}$ with an average mixing ratio of $0.5^{+0.8}$ ppm (there are no good data below $8 \mathrm{~km}$ ). The observations are performed in equatorial spring conditions. The altitude distribution of formaldehyde reveals correlation with the permanent haze opacity.
\end{abstract}

\section{Introduction}

The Martian atmosphere was studied with the Auguste spectrometer during the Phohos mission using the solar occulation technique. The instrument incorporated an ultraviolet (u.v.) spectrometer $(215-328 \mathrm{~nm}$ ). a FabryPerot interferometer for $i=762$ and $936 \mathrm{~nm}$ channels and an infrared (i.r.) spectrometer for two narrow spectral ranges centered at 3.7 and $1.9 \mu \mathrm{m}$ in the first and second orders of diffraction, respectively. The description of the instrument and the data collected with the u.v. spectrometer and interferometer are discussed by Blamont e' $a$ l. (1991): the preliminary analysis of the i.r. spectrometer data is presented by Krasnopolsky et al. (1989).

The principal objective of the i.r. part of the experiment was the simultaneous observation of the $\mathrm{HDO}$ and $\mathrm{H}_{2} \mathrm{O}$ band absorptions at $2720 \mathrm{~cm}^{-1}(3.7 \mu \mathrm{m})$ and $5340 \mathrm{~cm}^{-1}$ $(1.9 \mu \mathrm{m})$. The determination of the vertical profile of the

Correspendence to: O. I. Koriblev water vapor mixing ratio is discussed in a paper by Krasnopolsky et al. (1991), hereafter referred to as WVP. The data of the first order $(3.7 \mu \mathrm{m}$ region) were strongly affected by instrumental effects and were used for obtaining information on continuous absorption. which led to conclusions about the properties of dust (Korablev et al.. 1993). Here we present a further analysis of the data in the $3.7 \mu \mathrm{m}$ range obtained after incorporating appropriate instrumental corrections.

The problems with the Sun-tracking system prevented the u.v. spectrometer line of sight from reaching altitudes lower than $30-40 \mathrm{~km}$. The relative offset of the i.r. optical axis. however. allowed us to probe down to $8-10 \mathrm{~km}$. Nine successful observations in the i.r. were completed at sunset from 20 February to 26 March 1989 , from a circular orbit with a radius of $9670 \mathrm{~km}$ and spacecraft to limb distance of $9050 \mathrm{~km}$. The latitudes of the tangential point within this period varied from -3 to 20 , and the aerocentric solar longitude $L$, which is a measure of the Martian seasons varied from 1.5 to 18 , i.e. the observations were performed at the beginning of the Martian spring in the equatorial latitudes. The relevant information about the observational sequences is summarized in Table 1 .

Table 1. Observational sequence information

\begin{tabular}{rrrrrrr}
\hline Sequence & Date & U.T. & \multicolumn{1}{c}{$L_{\text {s }}$} & $\phi$ & $\begin{array}{c}\text { Longitude } \\
\text { at sunset }\end{array}$ \\
\hline 2212211 & 20.02 .89 & $16: 36: 58$ & 1.6 & -3 & 55.2 \\
2360639 & 7.03 .89 & $01: 04: 24$ & 8.7 & 6.8 & 44.25 \\
2362251 & 7.03 .89 & $17: 17: 20$ & 9.0 & 7.2 & 280.8 \\
2412311 & 12.03 .89 & $17: 36: 55$ & 11.6 & 10.6 & 237.45 \\
2440723 & 15.03 .89 & $01: 48: 24$ & 12.8 & 12.2 & 337.65 \\
2441524 & 15.03 .89 & $09: 50: 03$ & 13.0 & 12.5 & 94.95 \\
2450728 & 16.03 .89 & $01: 53: 30$ & 13.2 & 12.9 & 329.25 \\
2510753 & 22.03 .89 & $02: 19: 20$ & 16.1 & 17.0 & 277.35 \\
2560316 & 26.03 .89 & $21: 41: 24$ & 18.5 & 20.3 & 161.1 \\
\hline
\end{tabular}

Note. The sequence identification designates spacceraft time in days, hours and minutes, c.g. $221^{\mathrm{d}} 22^{\mathrm{h}} 1 \mathrm{I}^{\mathrm{m}}$. $L_{\mathrm{s}}$ is acrocentric solar longitude : $\phi$ is latitude 


\section{The instrument, and data reduction}

The i.r. spectrometer and its calibration are discussed in WVP; certain details. relevant to instrumental effects. are presented here. A common telescope and Sun-tracking system were used for the entire Auguste spectral unit. The instrument pointing system provided tracking of the solar disk center with an accuracy of $30 \mathrm{sec}$ arc outside the atmosphere: tracking in the dusty atmosphere suffered from an error in the pointing software. The Sun-tracking and related problems are considered in papers by Blamont ct al. (1991) and Chassefiere et al. (1992). The telescope is a $50 \mathrm{~mm}$ Cassegrain type with a focal length of $750 \mathrm{~mm}$. To avoid the need for beamsplitting. the fields of view of each of the spectrometers were offset from each other. The field of view of the i.r. spectrometer was designed to be offset by $8.6 \mathrm{~min}$ arc from the solar disk. but the pointing problems mentioned above led to an increase of this offset and consequently only $\approx 70 \%$ of the aperture was illuminated (see WVP and Chassefierc $e t$ al. 1992). The spectrometer field of view is $1.5 \mathrm{~min}$ arc which corresponds to a spatial resolution of $\approx 4 \mathrm{~km}$ at the limb from a given orbit.

An intermediate lens and a system of mirrors form an image of the Sun on the instrument slit. The slit of $110 \mu \mathrm{m}$, a toroidal grating with 300 lines per $\mathrm{mm}$ and a detector are placed on a Rowland circle with a radius of $83 \mathrm{~mm}$. A mechanical chopper of $2.5 \mathrm{kHz}$ frequency for synchronous detection is placed before the slit. The electrically cooled 48 element $\mathrm{PbSe}$ photoresistor detector array has 16 pixels in the first-order spectrum, covering the spectral range of $2740-2707 \mathrm{~cm}^{-1}(3.7 \mu \mathrm{m})$, and 20 pixels in the secondorder spectrum with a range of $5372-5292 \mathrm{~cm}^{-1}(1.9$ $\mu \mathrm{mi})$. Pixel size is $140 \times 125 \mu \mathrm{m}$ with a $10 \mu \mathrm{m}$ offset. The separation of diffraction orders was provided by filters placed just above the detector sapphire window. In order to avoid stray light problems and mixing of the diffraction orders, the joint between the two filters was shielded by a black material that resulted in 12 central pixels being out of use.

The instrument's resolving power calculated theoretically, taking into account the slit width. is $\lambda \Delta \lambda=1870$. Chromatic aberration in the optical path (see WVP) and an aberration of the toroidal grating reduce this value to $\approx 1700$. Since the width of a pixel is close to that of the slit. a spectrum focused at the detector has a spatial resolution of the same order as the pixel width $(0.125 \mathrm{~mm})$. Hence. the pixel function should be studied carefully. To do this we performed laboratory microprobe measurements which provided a chart of the sensitivities in different points of the pixel for a detector of the same type. Batsed on these data we assumed the pixel spectral response function to be a trapezoid with a baseline of $0.9 \Delta \lambda$ and a top of $0.8 \Delta i \ldots$ where $\Delta z$ is the average width of the pixel. Thus there is significant separation between the spectral response of adjacent pixels. which may cause reduction and even loss of narrow spectral features located between the pixels. The resulting resolving power of the instrument, taking into account the detector geometry and the sampling problem. is found to be of the order of $\approx 1300$.

In calibrations done at ground level. the instrument was adjusted so that the HDO absorption feature $\left(2720 \mathrm{~cm}^{-1}\right)$ was located at pixels 7-8 (see WVP, Section 3). It results in a dispersion relationship

$$
v_{n}=k\left(2736.0-2.044 n+0.002 n^{2}\right) \mathrm{cm}^{-1},
$$

where $k$ is the diffraction order, $n$ is the pixel number and $v_{n}$ corresponds to the center of the pixel. A small displacement of the grating during the launch was suspected by WVP, hence the constant term in equation (1) was corrected to be 2740.75 for measurements from Martian orbit. according to the position of the $\mathrm{CO}_{2}$ absorption peak. Updated studies of the second-order spectra considering the sampling problem and the individual nonlinearity of pixels, which affects the shape of the $\mathrm{CO}_{2}$ absorption feature. show that the correction of the constant term should be smaller (and closer to calibration data):

$$
v_{n}=k\left(2738.25-2.044 n+0.002 n^{2}\right) \mathrm{cm} \quad \text {. }
$$

The difference between equation (2) and the relationship given in WVP is 1.2 pixels. Fortunately this difference does not significantly affect the appearance of spectra near pixels 31,32 used in WVP for the determination of the water vapor mixing ratio profile; the conclusions of the WVP are thus still valid.

The main defects of the spectra observed in the $3.7 \mu \mathrm{m}$ region which need correction are the diversity in nonlinearity of individual pixels and a periodical distortion of spectra with a period of three pixels associated with the variation in detector temperature.

Due to aerosol extinction, the measured flux decreases during the occultation sequence. Thus, the nonlinearity of the detector and, primarily, the diversity of nonlinearity in individual pixels prevents reliable analysis of weak relative absorptions. The nonlinearity of the PbSe detector was studied during the ground calibrations of the spectrometer. These measurements allowed us to approximate the mean nonlinearity using only two parameters, with a power law providing the best fit (see Korablev et al., 1992). No credible data on the nonlinearity of each particular pixel were available. Certain observational sequences with highly disturbed Sun-tracking indicate an increase in the measured flux by a factor of 1.6. During these sequences the spectrometer field of view entirely encompassed the solar disk and an unattenuated flux was monitored. Comparing this flux with that observed with normal Sun-tracking we obtain an in-flight calibration. Resulting corrections for the various signal attenuations are presented in Fig. 1 : the details are given in Appendix A.

Figure 2 shows five occultation spectra of 12 March 1989 ; it shows raw data and the effect of corrections. A transmission interval chosen for illustration purposes in this paper corresponds to a part of the occultation curve where the spectral features can be distinguished, the effect of introduced corrections is significant. and signal to noise ratio is reasonably good.

The data corrected for the individual pixel nonlinearity exhibit periodical structure (Fig. 2b). It might seem surprising, because the nonlinearity correction (see Fig. 1) shows no evidence of periodicity. However, the periodical deformation with a period of three pixels can always be seen when the detector temperature of the measured spec- 


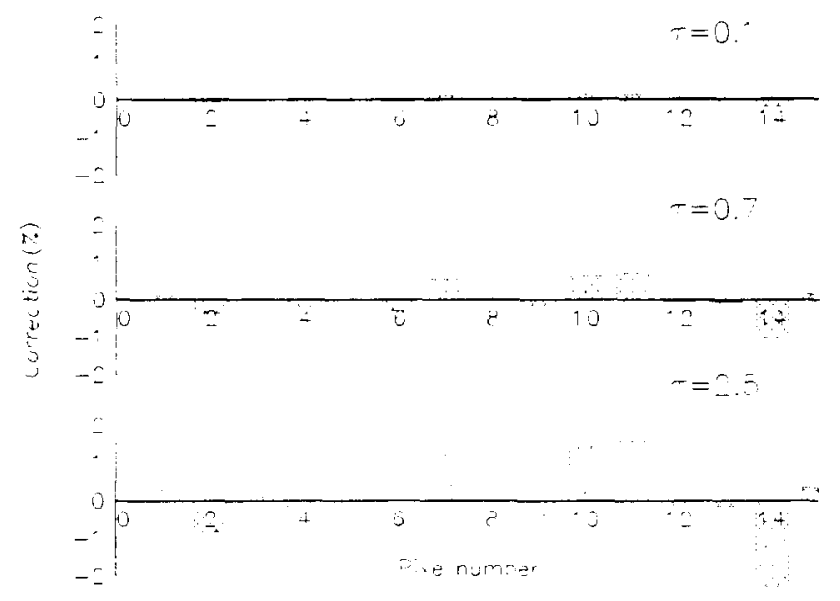

Fig. 1. Nonlinearity corrections for each pixel in the first diffraction order for three values of signal attenuation ( $\tau$ is the horizontal optical depth)

trum differs from that of the reference one. The nature of the periodical deformation of the first-order spectra is still not finally understood. No atmospheric constituent within the given spectral range shows evidence of this periodicity. The effect reveals an apparent correlation with the temperature of the detector; however, the relationship is complicated. The cooling of the sensitive elements is provided by a three-stage Peltier-type cooler down to a temperature that is $100 \mathrm{C}$ below that of the instrument $(\approx 20 \mathrm{C})$. No means of temperature stabilization was available. so that during the short ( $10-15 \mathrm{~min})$ periods of observations when the instrument was switched on. a significant change in the temperature of the detector occurred. The duration of an oscillation sequence at the Martian orbit was typically less than $30 \mathrm{~s}$ (tangential descent velocity of $\approx 2 \mathrm{~km} \mathrm{~s}^{-1}$ ), and heating of the sensitive elements during this period anounted to $0.1-0.3 \mathrm{C}$.

The electrical system of the i.r. spectrometer includes 48 channel amplifiers multiplexed by three groups of 16 channels each, distributed over three hybrid units. Each group is equipped with a synchronous detector and integrator. The arrangement of circuits connecting the detector and the amplification units resulted in the signal from each of three subsequent pixels being accumulated simultaneously, i.e. the measurement of a spectrum begins with pixels $1-3$, then $4-6$, etc.

The origin of the periodical deformation is associated with the use of three different synchronous detectors for the subsequent pixels: certain properties of the $\mathrm{PbSe}$ detector should also contribute. The temperature trend leads to the change of the resistance of pixels and. then. to phase shifts in the entry preamplifier's circuits. The shifts affect the three synchronous detectors which are not perfectly identical, and the sensitivities of the subsequent measuring channels are hence slightly different. It is not clear why the periodical distortion is significant for the first order of diffraction. but it is weaker by two orders of magnitude in the second order. The flux at the detector is almost equivalent for both diffraction orders and the preamplifiers used for the two parts of the detector are the same.

For the case shown in Fig. 2, three-period distortion is unexpectedly concealed by the pixels nonlinearity. The amplitude of this error is of the same order as the effect of nonlinearity and it is necessary to correct it also. The description of the correction algorithm is given in Appen$\operatorname{dix} B$.

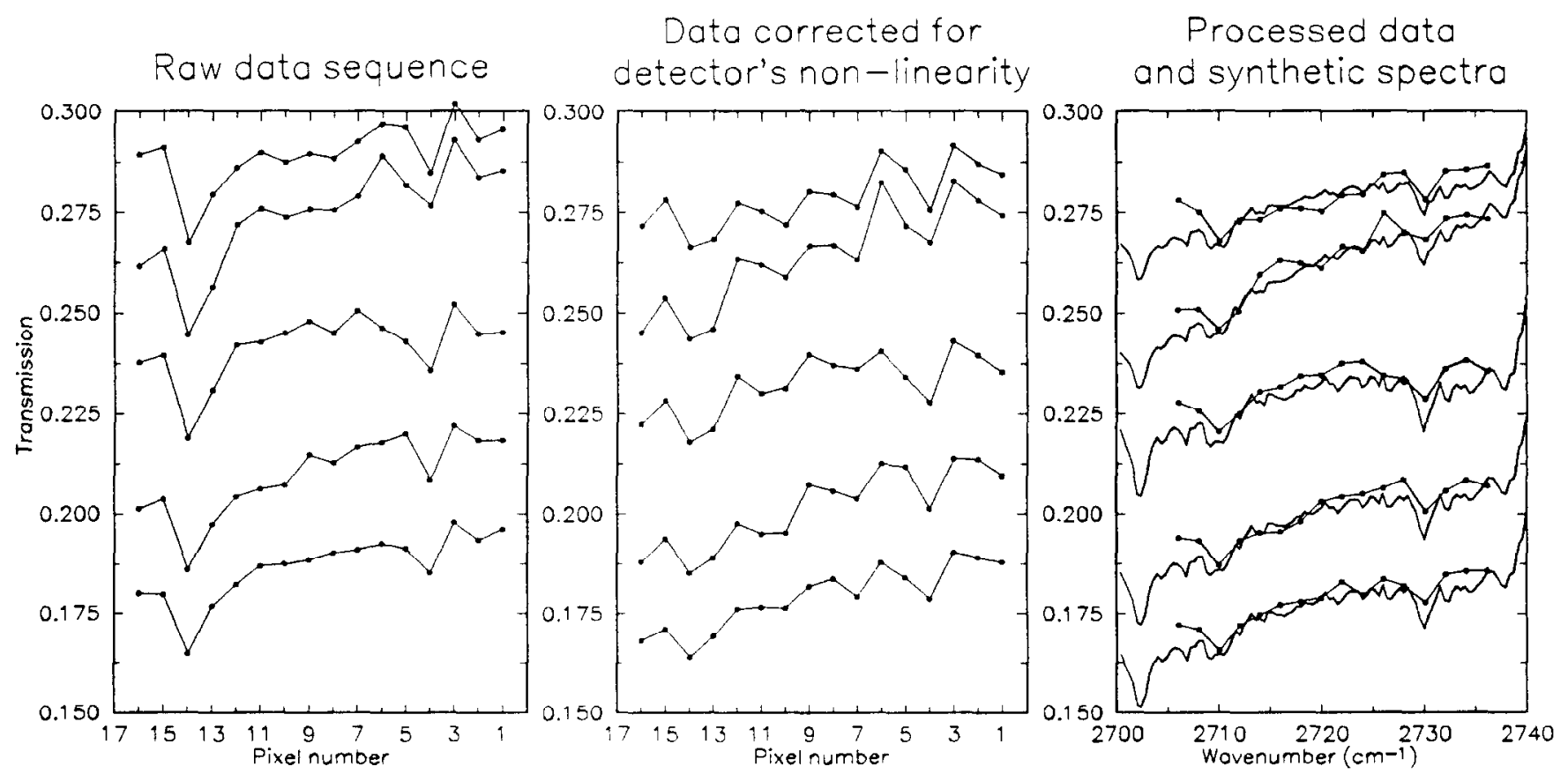

Fig. 2. Five spectra observed in sequence on 12 March 1989 (2412311) in the transmission interval from 0.15 to 0.3 demonstrating the raw data (left), the data corrected for the individual nonlinearity of pixels (center) and data corrected to remove periodical deformation of spectra with corresponding synthetic spectra of formaldehyde (right) 


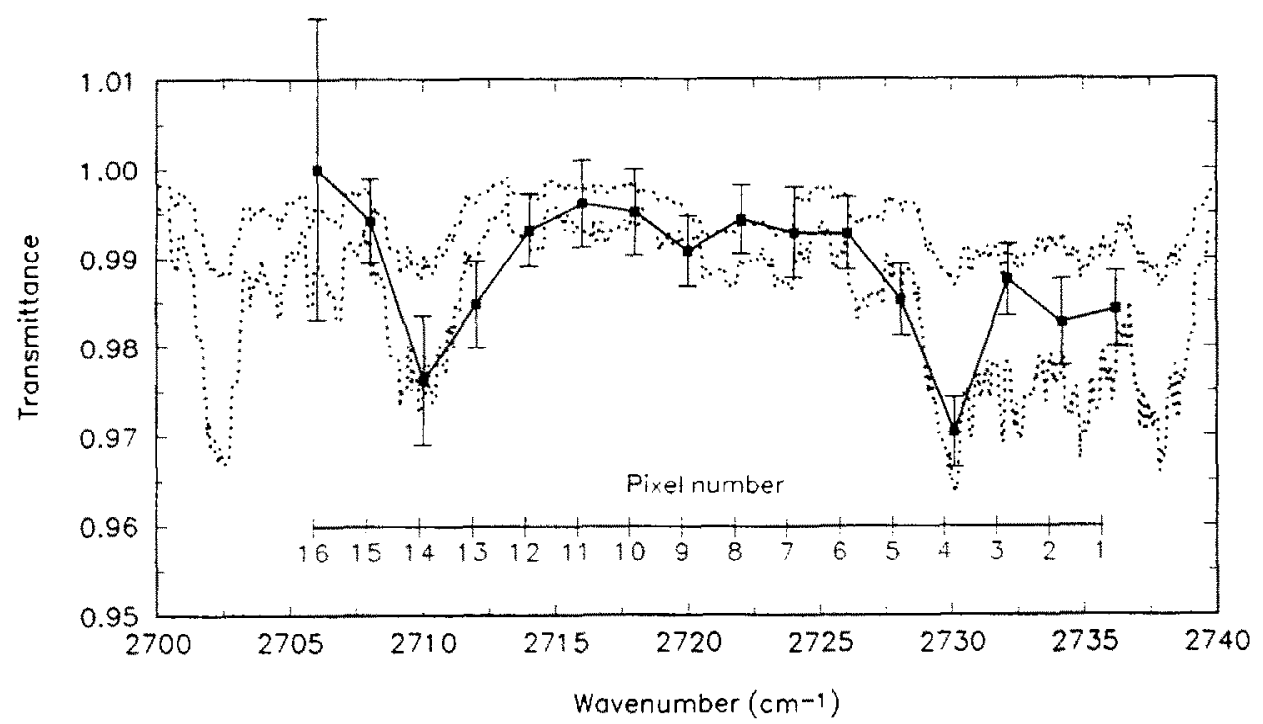

Fig. 3. The mean spectrum transmittance (solid line) in the range of $3.7 \mu \mathrm{m}$ is displayed with two synthetic spectra computed for $\mathrm{CH}_{2} \mathrm{O}$ mixing ratios of 0.3 and $1 \mathrm{ppm}$ (dotted curves) vs wavenumber. The error bars are one- $\sigma$

\section{The average spectrum}

The average first-order spectrum obtained after a set of corrections described above is presented in Fig. 3. It is a result of averaging of the nine successful observational sequences $(\sim 100$ spectra) after appropriate corrections have been applied (see Section 2). We have done no preliminary selection of spectra; however. the beginning and the end of an occultation were removed in order to increase the signal to noise ratio. Each spectrum is also corrected to remove continuum absorption and normalized to unity. We assumed that the continuum absorption was described by a straight line within the given spectral range. The mean tangential altitude is $\approx 17 \mathrm{~km}$. The error bars of the mean spectrum include random noise of the detector $\left(\approx 0.2^{0}\right)$ and the residual uncertainties of corrections which are collectively estimated to be $\approx 1 \%$ (see Appendices $A$ and $B$ ). The correction errors are not the same for different pixels.

The mean spectrum shown in Fig. 3 demonstrates two main features: an absorption above $2729 \mathrm{~cm}^{1}$ and an absorption at $2710 \mathrm{~cm}$. These fcatures can be found readily in almost all the spectra measured below $20 \% 25$ $\mathrm{km}$. Formaldehyde may be considered as a potential candidate to explain these absorption leatures.

A number of strong $\mathrm{CH}_{2} \mathrm{O}$ lines (intensities $~-10^{\text {ty }}$ $\mathrm{cm}{ }^{2}$ molec ' $\mathrm{cm}$ ') can be found in the spectroscopic data banks, e.g. HITRAN near 2710 and below $2730 \mathrm{~cm}$ ' (Rothman at al. 1987). Calculations were first done using the HITRAN database. and integrating along the path of several atmospheric layers (so as to reproduce the geometrical conditions of observations). and taking into account the profile and overlapping of spectral lines.

Though the positions of two of the most distinct features in the observed mean spectrum agree with those in the synthetic spectrum. the shape of the mean spectrum differs from the modeled one. Laboratory spectroscopy measurements by Nakagawa of al. (1976) indicate that a number of weak $\mathrm{CH}_{2} \mathrm{O}$ lines in the region close to 2700 $\mathrm{cm}^{-1}$ are not consistent with those given in the HITRAN database [the data of GEISA (Chedin ef al. 1986) and HITRAN databases almost coincide]. Our study of this problem by means of laboratory Fourier spectroscopy at atmospheric pressure confirmed this discrepancy. The investigation of the spectrum of formaldehyde is troublesome because of its tendency to easy polymerization. The most thorough analysis of the formaldehyde i.r. spectrum was carried out by Brown and Hunt (1978) and Brown et al. (1979). The spectral line parameters tabulated in these works also reveal a discrepancy with the databases. A large number of weak lines corresponding to high rotational states is missing in the databases, e.g. within the range of our interest $\left(2710-2730 \mathrm{~cm}^{-1}\right)$ HITRAN indicates $\approx 80$ lines of the $\approx 350$ lines indexed by Brown et al. (1979). Thus we used the data from Brown et al. (1979) for the smmulation of the synthetic spectrum. The missing spectral lines in the databases led to an overestimation of formaldehyde abundance in our earlier presentations (Korablev et al.. 1992).

Two synthetic spectra computed with the data from Brown of al. (1979) are shown in Fig. 3 along with the observed mean spectrum. They reflect the mixing ratios of $\mathrm{CH}, \mathrm{O}$ of 0.3 and $1 \mathrm{ppm}$ and they are for a tangential altitude of $17 \mathrm{~km}$. corresponding to the average height of the mean spectrum. The synthetic spectra match the measured mean spectrum reasonably well, excluding, however, the spectral region above $2730 \mathrm{~cm}^{\prime}$ ' (pixel 3), perhaps due to the still-questionable synthetic spectrum.

The modeling of the synthetic spectra was done by applying an atmospheric model with temperatures lower than those in the COSPAR Reference Atmosphere (Seiff. 1982). We assumed the following temperature profile based on the water vapor profile described in WVP: $T(z=0 \mathrm{~km})=210 \mathrm{~K}, T(25 \mathrm{~km})=170 \mathrm{~K}$ and $T(50 \mathrm{~km})$ $=147 \mathrm{~K}$ (the gradient is constant between the indicated points). The infinite resolution spectra computed are 
then convolved with the function of spectral response of the instrument. including both the resolution of the spectrometer and the pixel spatial response as discussed in Section 2 .

A weak absorption at $2720-2723 \mathrm{~cm}^{-1}$ exhibited in the synthetic spectrum is due to the HDO absorption band $3.68 \mu \mathrm{m}$. The mean spectrum gives infirm evidence of this band at $2720 \mathrm{~cm}^{\prime}$; the absorption. however, is less than $0.5 \%$, which is within the error hars of the mean spectrum. Sometimes it is more prominent in individual spectra. Low absorption in the HDO band observed in the first order of diffraction is in accord with very dry conditions in the equatorial atmosphere. The synthetic spectrum is computed for the water vapor profile measured in the second order of diffraction (WVP) and the $\mathrm{D} / \mathrm{H}$ ratio equal to $8 \times 10^{+}$(Bjoraker et al. 1989). A number of weak formaldehyde lines in the region $2715-2725 \mathrm{~cm}$ ' could also mash the HDO absorption leature. Therefore, our measurements can only provide an upper limit of HDO abundance, giving $\mathrm{D} H<5 \times 10^{+}$, that is, close to the value of $6 \times 10^{+}$obtained by Owen et al. (1988) and to the Kuiper Airhorne Ohservatory measurements (Bjoraker et (1). 1989).

Methane $\left(\mathrm{CH}_{4}\right)$ has a system of nearly equidistant absorption features in our spectral range. The upper limit of methane is $3.7 \mathrm{ppm}$ (Horn et al. 1972) and results in an absorption $\sim 0.5 \%$. The distance between the $\mathrm{CH}_{4}$ features at 2712 and $2727 \mathrm{~cm} \quad$ is lower than that between the observed features: hence. $\mathrm{CH}_{+}$cannot be al candidate to explain them. Other minor constituents of the Martian atmosphere with absorption bands in the spectral range in question, such as $\mathrm{O}_{3}, \mathrm{~N}, \mathrm{O}$ and $\mathrm{CO}_{2}$ isotopes. have no distinct features.

\section{Vertical distribution}

Although the individual spectra do not have large signal to noise ratios. it is still possible to make an estimation of the absorption at different altitudes. assuming formaldehyde to be the absorber. A simple wo-dimensional synthetic model is constructed to study the altitude distribution. The $\mathrm{CH}_{2} \mathrm{O}$ mixing ratio is alssumed to be constant with altitude. To determine the altitude we used the altitude vs time dependence suggested by Blamont at al. (1991). modified below $\approx 15 \mathrm{~km}$ as discussed in WVP. The synthetic spectral of atmospheric absorption were computed as discussed in Section 3. The set of synthetic spectra related to different altitudes and $\mathrm{CH}_{2} \mathrm{O}$ mixing ratios is generated to obtain a spectrum which best fits the observed spectrum. This approach provides simultaneous reduction of the occultation protiles. The slope in observed spectrat (see Fig. 2) is due to the continuous absorption caused by aerosols. It is fitted using a straight line which is described by two additional free parameters in the model.

Four profiles obtained using the above method are shown in Fig. 4. Significant features attributed to $\mathrm{CH}_{2} \mathrm{O}$ can only be measured below an altitude of $20 \mathrm{~km}$. The upper point of the identification of the absorptions is close to the upper boundary of the permanent haze layer. Moreover, a correlation can be found between the formaldehyde mixing ratio and slant optical thickness profiles.

For the long path provided by the occultation geometry, the strongest $\mathrm{CH}_{2} \mathrm{O}$ rovibrational transitions, which might be responsible for the features near $3.7 \mu \mathrm{m}$. should be saturated and overlap one another. However nonsaturated wings of the lines are mainly still of the Doppler shape. Thus, the curve of growth of absorption in a spectral channel which covers up to dozens of lines flattens for formaldehyde mixing ratios more than $-3 \mathrm{ppm}$. Therefore. any small spectrum deformation due to noise or systematic errors might significantly affect the retrieved mixing ratio. Consequently. $\mathrm{CH}_{2} \mathrm{O}$ mixing ratios which exceed the level of $\approx 3 \mathrm{ppm}$ are less reliable.

The error bars of the profiles correspond to a one- $\sigma$ confidence level. For certain measurements. only lower or upper limits are available. The evaluation of the error bars includes the above-mentioned random noise of the detector and the residual correction errors. The relative uncertainty of $12 \%$ in absorption for the simultaneous fit of all 16 pixels considered results in uncertainty of up to an order of magnitude for the mixing ratio.

In all, eight altitude profiles of $\mathrm{CH}_{2} \mathrm{O}$ are available. The sequence 2360639 (Fig. 4b) shows an increased formaldehyde amount in comparison with the others. The sequence 2362251 is influenced strongly by the Sun-tracking perturbations, and the handling of individual spectra is not possible in this case.

The mixing ratios of formaldehyde retrieved with the above assumptions. along with the lower and upper limits, are plotted in Fig. 5a. The average lower and upper limits are shown in Fig. 5b. The atmospheric absorber is thus detected mainly below $25 \mathrm{~km}$ with levels of $0.1-2 \mathrm{ppm}$. The average profile changes from $\approx 0.15 \mathrm{ppm}$ at $25 \mathrm{~km}$ to $\approx 1 \mathrm{ppm}$ below $15 \mathrm{~km}$. The lowest altitude of sounding is $\approx 8 \mathrm{~km}$.

\section{Discussion}

Assuming formaldehyde to be responsible for the discussed absorption features, the vertical integration of the profiles retrieved in eight occultation sequences results in the column densities of $\mathrm{CH}_{2} \mathrm{O}$ given in Table 2. The mixing ratios averaged over the altitude range above the lowest sounded altitude are also presented. From Table 2 the mean column density of $\mathrm{CH}_{2} \mathrm{O}$ is found to be $\left(3.5^{+10}=10^{16} \mathrm{~cm}\right.$ = with the mean mixing ratio above 8 $\mathrm{km}$ of $0.5^{+}+1.8 \mathrm{ppm}$. The error bars are computed by height integration of the average lower and upper limit profiles (Fig. 5b). A strong asymmetry of the error bars is caused by the nonlinearity of the curve of growth for saturated lines as discussed in Section 4.

Beer et al. (1971) measured the spectra of Mars from the McDonald Observatory in the range $2500-3500 \mathrm{~cm}$. with a $0.58 \mathrm{~cm}$ 'resolution and found, in some cases. unidentified spectral features. Adequate spectroscopic databases did not exist 20 years ago. and the nature of these features was not understood. Comparing Beer's mean spectrum of Mars with the synthetic spectra computed for a combined effect of absorption in the terrestrial 

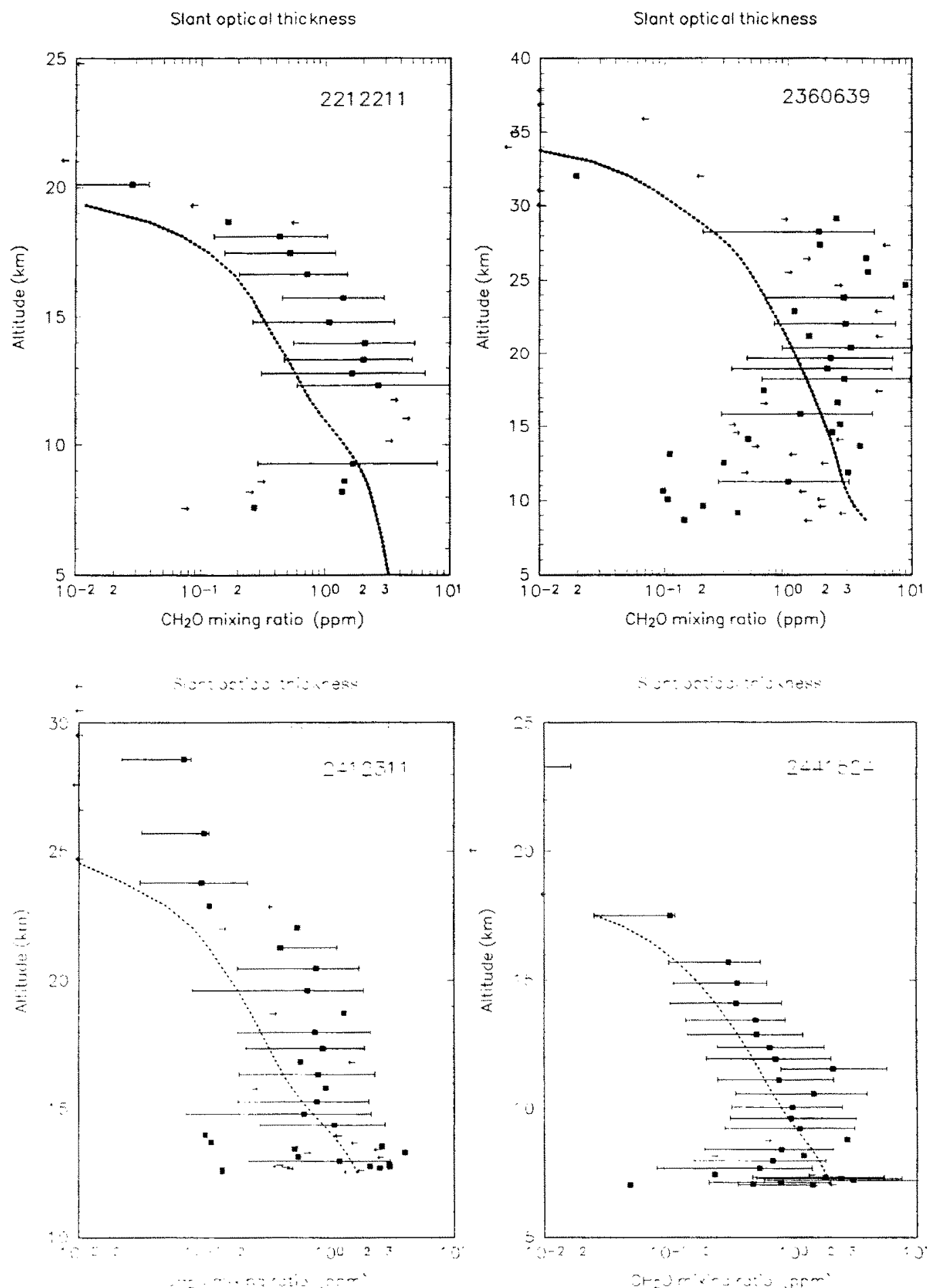

Fig. 4. The formaldehyde volume mixing ratio $(f(H, x)$ measured in four observational sequences vs altitude. The squalen represent the results of fitting the observed corrected spectrat with the $\mathrm{CH}_{2} \mathrm{O}$ synthetics ats shown in Fig. 20. The crror bars are one- $\pi$. The arrows have the same sense as error hars. when only the kower or upper limits are avalable. The vertical bar shows the instrument spatial resolution (FWHM). The horizontal optical depth profiles $\left(t_{\text {dam }}\right)$ are also shown (dotted curves) at the same scale

and Martian atmospheres and lor a formaldehyde-containing Martian atmosphere (sce Fig. 6). we can conclude that some of Beer's unidentified features could be attributed to $\mathrm{CH}_{2} \mathrm{O}$. Comparison with the synthetic spectra can give a rough estimate of the formaldehyde mixing ratio as $\sim 0.3 \mathrm{ppm}$.
According to Owen and de Bergh (personal communication. 1992) the set of data obtained at Mauna Kea $3.6 \mathrm{~m}$ telescope in a wide spectral range near $3 \mu \mathrm{m}$ with a resolution of $0.03 \mathrm{~cm}$ ' shows no evidence of formaldehyde. The upper limit is $0.3 \mathrm{pmm}$. which is in reasonable agreement with our data. However, the lines of HDO 



Fig. 5. Composite plot lor all the eight successful sequenees (left). Average height profiles of lower and upper limits (right)

absorption band $3.68 \mu \mathrm{m}$ which are not observed in our data (see Section 3) are clearly seen in the data by Owen et al. (1988). The mean water vapor abundance is $\approx 10$ precipitated $\mu \mathrm{m}$ for near-equatorial and mid-latitudes (Jakosky, 1985). Owen of al. (1988) found from their data the value of 5 prec. $\mu \mathrm{m}$ of $\mathrm{H}_{2} \mathrm{O}$ on Mars. Our measurements in the second order of diflraction $\left(1.87 \mu \mathrm{m} \mathrm{H}_{2} \mathrm{O}\right.$ band) resulted in a vertical profile of water vapor in the altitude range of $10-40 \mathrm{~km}$. If we assume $\mathrm{H}_{2} \mathrm{O}$ is well mixed below $10 \mathrm{~km}$ (Davies. 1979). then the altitude profile from WVP yields an abundance of $\delta$ prec. $\mu \mathrm{m}$. However. above $10 \mathrm{~km}$ we observed a steep decrease of the water vapor profile. The abundance of $H, O$ calculated with our altitude profile varies from (1.t pree. $\mu \mathrm{m}$ above $15 \mathrm{~km}$ to 0.1 prec. $4 \mathrm{~mm}$ abose $20 \mathrm{~km}$. Thus, the mean first-order spectrum (Fig. 3) corresponds to extremely

Table 2. Total column values of lomaldehyde obtained by height integration of its profiles abowe the altitude $z_{\ldots . .1}$. Which is the low border of sounding. Mixing ratios of $\mathrm{CH}, \mathrm{O}$ computed above this altitude are also indicated inurlice pressure supposed to be 6 mbar)

\begin{tabular}{|c|c|c|c|c|}
\hline Sequence & $\begin{array}{l}z_{1, k,} \\
(\mathrm{~km})\end{array}$ & $\begin{array}{l}\text { Column dens } \\
(\times 1)^{1:} \mathrm{cm}\end{array}$ & is & $\begin{array}{c}\text { Mixing ratio } \\
\text { (ppm) }\end{array}$ \\
\hline 2212211 & 14 & 2.9 & & 0.6 \\
\hline 2360639 & 10 & 13.1 & & 1.7 \\
\hline 2412311 & 14 & 1.9 & & 0.5 \\
\hline 2440723 & 11 & 1.7 & & 0.2 \\
\hline 2441524 & 8 & 4.5 & & 0.5 \\
\hline 2450728 & 11 & 2.1 & & 0.3 \\
\hline 2510753 & 14.5 & 0.35 & & 0.1 \\
\hline 2560316 & 13 & 0.5 & & 0.15 \\
\hline
\end{tabular}

dry atmospheric conditions and our observations did not detect HDO.

Formaldehyde also exhibits an absorption band system in the u.v. with continuous absorption between 260 and $360 \mathrm{~nm}$. Assuming an absorption cross-section. $\sigma=$ $2.6 \times 10^{-10} \mathrm{~cm}^{2}$ at $300 \mathrm{~nm}$ (DeMore et al. 1987), an absorption of $1.5 \%$ results for the quantity of $\mathrm{CH}_{2} \mathrm{O}$ deduced in this paper, taking an air mass factor of 3 for observations from the Earth. Therefore formaldehyde is not expected to be detected by u.v. spectrometers which have been used to study Mars. Likewise, the u.v. part of the Auguste solar occultation spectrometer would also miss the $\mathrm{CH}_{2} \mathrm{O}$ detection, because u.v. meisurements were not performed at altitudes lower than $\approx 35 \mathrm{~km}$.

The mechanism responsible for the formation of $\mathrm{CH}_{2} \mathrm{O}$ on Mars needs investigation. Next, we just summarize our present understanding of the problem. It is surmised that the early Earth atmosphere which is in some sense similar to the present atmosphere of Mars should contain formaldehyde as its most abundant trace constituent (BarNun and Chang, 1983). In the present Earth's atmosphere. formaldehyde is formed readily on oxidation of methane. The rate constant of the process is approximately $7 \times 10^{15} \mathrm{~cm}^{3} \mathrm{~s} !$. In addition to its loss due to photolysis, formaldehyde can also be removed by $\mathrm{OH}$ radicals. with a rate constant of about $10^{14} \mathrm{~cm}^{3} \mathrm{~s}$. Although methane has not yet been detected on Mars, and conventional models do not predict it, it is nevertheless instructive to estimate the required methane abundance for the observed $\mathrm{ppm}$ level of formaldehyde. The simple pathway just described would require methane of the order of $1 \%$. a quantity too large to have escaped detection so far. We conclude, therefore, that some other mechanism must be 


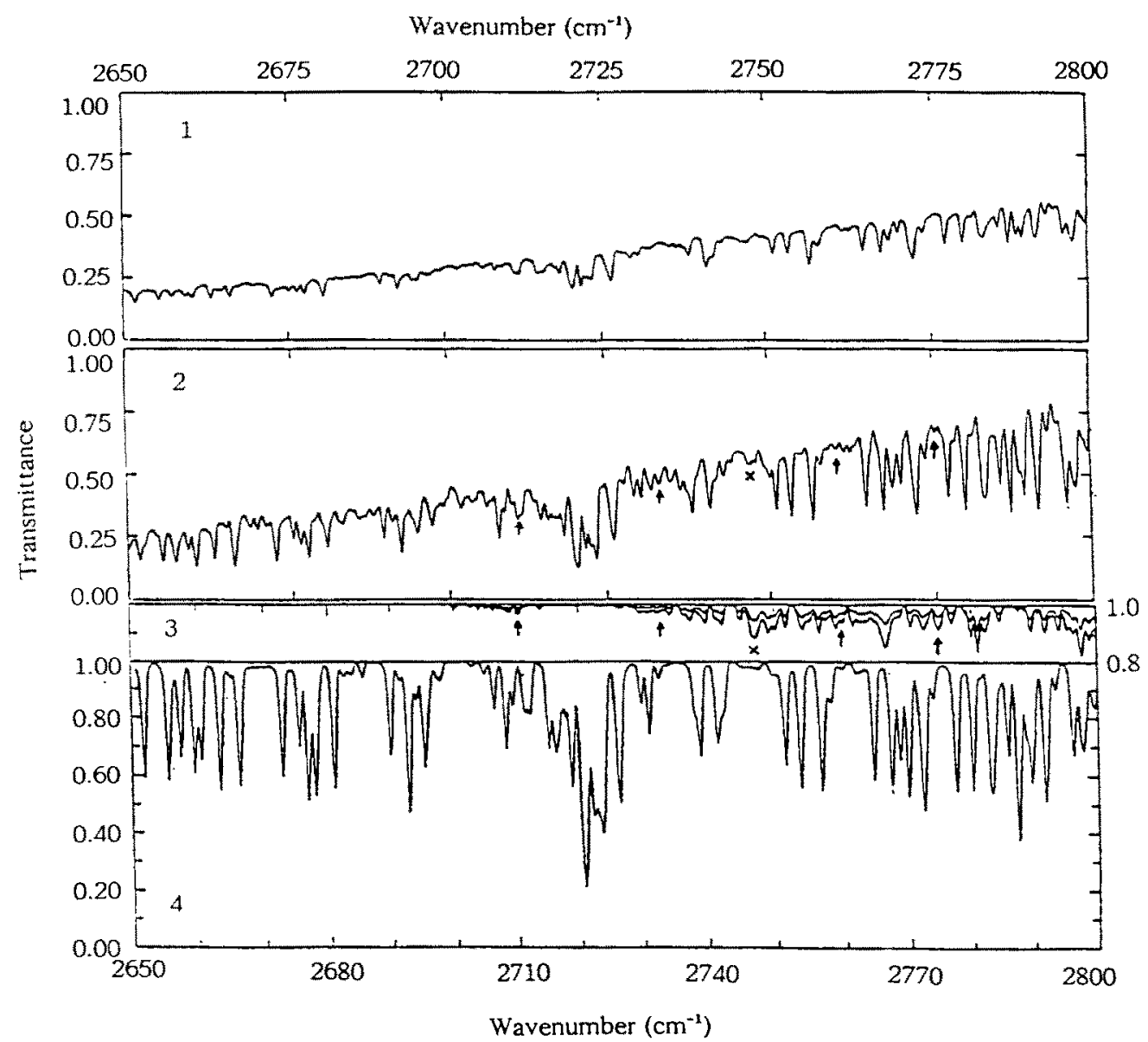

Fig. 6. A spectrum of the Martian atmosphere observed from the Earth by Beer et al. (1971) (curve 2) with the corresponding reference spectrum (curve 1). In the same scale the synthetic spectra of extra-terrestrial formaldehyde computed for mixing ratios of 1 and $10 \mathrm{ppm}$ and reproducing the observational conditions is presented (curve 3) with the synthetic spectra of the Earth's atmosphere to simulate more correcty the veferenee one (eurve 4). The arrows indicate the absorption features not identified by Beer $t$ al and. coinciding with that of $\mathrm{CH}_{2} \mathrm{O}$. the cross marks a strong feature at $2747 \mathrm{~cm}$ which can also be attributed to formaldehyde

responsible for the formation of lomaldehyde on Mars. The thermochemical equilibrium mixing ratio of $\mathrm{CH}_{2} \mathrm{O}$ is also small, about 10 ". With mixing ratio $f_{c 0}=$ $7 \times 10^{4}, f_{H}=5 \times 10^{5}$ and thermodynamic constants from Westley at al. (1991). Thermochemical equilibrium is, however, not a good assumption in the Martian atmosphere. Another possibility involves reactions which can possibly occur in the relatively dry atmosphere. Specifically the reactions are as hollows:

$$
\begin{aligned}
& \mathrm{H}+\mathrm{CO} \rightarrow \mathrm{HCO}+\mathrm{hu} \\
& \qquad k_{1}=2 \times 10^{13} \exp (-1366 \mathrm{~T}) \\
& \mathrm{HCO}+\mathrm{O}, \rightarrow \mathrm{CO}+\mathrm{HO}_{2} \cdot \\
& k_{2}=3.5 \times 10^{2} \exp (-140 \mathrm{~T}) \\
& \mathrm{HCO}+\mathrm{HO}_{2} \rightarrow \mathrm{CH}_{2} \mathrm{O}+\mathrm{O}_{2} \cdot \\
& k_{1}=5 \times 10^{\prime \prime}
\end{aligned}
$$

[rate constants are taken from Westley et al. (1991)].
Photolysis is the dominant loss mechanism for formaldehyde in the daytime on Mars. and the "average" rate of photolysis, $J$, is $2 \times 10^{5} \mathrm{~s}$, or an "average" photochemical lifetime of approximately $13 \mathrm{~h}$. With the above chemical pathways the following expression for the $\mathrm{CH}_{2} \mathrm{O}$ mixing ratio is obtained:

$$
f_{(\mathrm{H}, 0}=1 \cdot f f_{(0,} k_{1}[\mathrm{H}] /\left(1+k_{2}\left[\mathrm{O}_{2}\right] / k_{3}\left[\mathrm{HO}_{2}\right]\right)
$$

This expression yields $3 \times 10^{1}$ for the formaldehyde mixing ratio on Mars. This is far too low in comparison with the observed ppm level for this species. For photochemically active species, such as formaldehyde, however, the daytime average value calculated here may not be indicative of abundances throughout the day and night, and especially for the terminator to which the solar occultation meatsurements correspond.

Therefore. the simple chemical models could yield very low formaldehyde mixing ratios. However, more soph- 
isticated models might explain the observations by taking into account gas phase reactions only. According to a recent study by Moreau et al. (1992) the model. which is an improvement over their carlier two-dimensional model of the Martian atmosphere (Moreau el al., 1991) suggests that it is possible to maintain a steady-state mixing ratio of $0.1-0.2 \mathrm{ppm}$ in the Martian equatorial region. The model includes about 30 reactions responsible for organic chemistry, and accounts for dust suspended in the atmosphere which reduces the u.v. flux. The source of the formaldehyde formation in the model is methane. with the assumed mixing ratio at the surface of $3 \mathrm{ppm}$.

The altitude profiles show a strong correlation of $\mathrm{CH}_{2} \mathrm{O}$ with the permanent haze layer. Dust is therefore suspected to contribute to the formaldehyde formation. It might be shielding of the photolyzing $u . v$. radiation, or possibly the heterogeneous (gas-solid) chemistry. The u.v. irradiation should play a principal role in these interactions. The formation of complex organic compounds in the laboratory has been reported, e.g. according to Muchin (personal communication, 1991), laboratory experiments demonstrate the formation of $\mathrm{CH}_{2} \mathrm{O}$ on u.v. irradiation of a $\mathrm{CO}_{2}$ and $\mathrm{H}_{2} \mathrm{O}$ mixture in the presence of magnetite particles. conditions close to those in the Martian atmosphere.

Since our measurements are for the equatorial region. the planetary average mixing ratio of $\mathrm{CH}_{2} \mathrm{O}$ might be smaller by a factor of 3 or more. Temporal behavior of the formaldehyde abundance shows a considerable decrease by the end of the observational cycle. Thus, the higher values of $\mathrm{CH}_{2} \mathrm{O}$ at the ppm level (e.g. in sequence 2360639 ), may be related to the variability in dust opacity, solar activity, etc. It is thus conceivable that the abovementioned ground-based measurements averaged over the entire planetary disk could miss the detection of $\mathrm{CH}_{2} \mathrm{O}$.

Formaldehyde is a first step in the formation of complex organic compounds and its detection can be important for the problem of prebiotic chemistry on Mars. for the planning of future planetary spacecraft missions and future Earth-based spectroscopic observation of the red planet.

Acknowledgements. We thank Dider Moreat for fruitful discussions and timely information on the modeling results and Martin de Mazicre for suggesting a concept of synthetic spectra simulation taking the sampling problem into account. AR wishes to acknowledge the support provided by a grant from the American Astronomical Society. SA acknowledges support received from the NASA Solar Systom Exploration Division under grant NAGW-2561

\section{References}

Bar-Nun, A, and Chang, S., Photochemical reactions of water and carbon monoxide in Earth's primitive atmosphere. $J$. geophys. Res. 88, 6662-6672, 1983.

Beer, R., Norton, R. H. and Martonchik, J. V., Astronomical infrared spectroscopy with a Connes-type interferometer: II-Mars. 2500-3500 cm '. Icarus 15. 1-10. 1971

Bjoraker, G. L., Mumma, M. J. and Larson, H. P., Isotopic abundance ratios for hydrogen and oxygen in the martian atmosphere. Bull. Am. Astron. Soc. 21.990 (Abstract). 1989.
Blamont, J. E., Chassefiere, E., Goutail, J. P., Mege, B., NunesPinharanda, M., Soushon, G., Krasnopolsky, V. A.. Krysko, A. A. and Moroz. V. I., Vertical structure of dust and ozone in the martian atmosphere deduced from solar occuitation measurements. Planct. Space Soi. 39, 175-187. 1991.

Brown, L. R. and Hunt. R. H., The ground state of H.CO. $J$. Mol. Spece. 73, 277-289. 1978.

Brown, L. R., llunt, R. Il. and Pine, A. S., Wavclength. line strength, and assignment in the doppler-limited spectrum of formaldehyde from $2700103000 \mathrm{~cm}^{-1}$. J. Mol. Spec. 75. $406-428.1979$.

Chassefiere, E., Blamont, J., Krasnopolsky, V. A., Korablev, O. I., Atreya, S. K. and West, R. A., Vertical structure and size distributions of martian acrosols from solar occulation measurements. Karus 97, 46-69, 1992.

Chedin. A., Husson, N., Scott, N. A., Cohen-Hallaleh, I. and Berrior, A., The GEISA data bank 1984 version. Laboratoire de Meteorologie Dynamique du CNRS. Intemal note N127. 1986.

Davies, D. W., The vertical distribution of Mars water vapor. $J$. geophys. Res. 84. 2875.1979.

DeMore, W. B., Molina, M. J., Sander, S. P., Golden. D. M., Hampson, R. F., Kurylo. M. J.. Howard, C. W. and Ravishankara, A. R., Chemical kinetics and photochemical data for use in stratospheric modeling. JPL Publication no. 87-41, 1987.

Jakosky, B. M., The seasonal cycle of water on Mars. Space Sri. $\operatorname{Rer}, 41,131-200,1985$.

Horn, D., Mc Afee, J. M.. Winer, A. M.. Herr, K. C. and Pimental, G. C.. The composition of the martian atmosphere: minor constituents. Kcam 16, 543, 1972.

Korablev, O. I., Krasnopolsky, V. A. and Rodin, A. V., Vertical structure of martian dust measured by the solar occultation from the Phohos spacecraft. Leurus. in press, 1993.

Korablev, O. I., Ackerman, M.. Krasnopolsky, V. A., Moroz, V. I.. Muller, C. and Rodin. A. V.. Tentative identification of the formaldehyde in the martian atmosphere. Pros. XXIX COSPAR Mecing. Washington. D.C.. 199?.

Krasnopolsky, V. A., Moroz, V. I.. Krysko, A. A.. Korablev, O. I. Zhegulev, V. S., Grigoriev, A. V., Tkachuk, A. Yu., Parshev, V. A., Blamont, J. E. and Goutail. J. P., Solar occultation spectroscopic measurements of the Martian atmosphere at 1.9 and $3.7 \mu \mathrm{m}$. Nature. Lond. 341, 603-604, 1989.

Krasnopolsky, V. A., Korablev, O. I., Moroz, V. I., Krysko, A. A., Blamont, J. F. and Chassefiere. E.. Infrared solar ocultation sounding of the Mattian atmosphere from the Phohos spacecraft. Icarte 94. 32 44. 1991.

Moreau, D., Esposito, L. W. and Brasseur. G., The chemical composition of the dust-free martian almosplice: preliminary results of a two-dimensional model. I, grophlys. Res. 96. N B5, 7933-7945, 1991 .

Moreau, D., Falise, E., Muller, C., Rosenquist, J., Marten, A., Korablev, O. and Esposito. L. W., Organic chemistry in the current martian atmosphere: theoretical and experimental investigations. Bull. Am. Astrm. Sor. 24, N3 (Abstract), 1992.

Nakagawa, T., Yamada, K. and Kuchitsu, K.. Vibration rotation spectrum of formaldehyde $\mathrm{C}^{\mathrm{H}}$ streching fundamentals $v_{1}$ and y. J. Mol. Spece. 63, 485.508 .1976$.

Owen, T., Maillard. J.-P., de Bergh, C. and Lut\%, B. L., Deuterium on Mars: the abundance of $\mathrm{HDO}$ and the value of D.H. Scicnce 240, 1767-1770. 1988.

Rothman, L. S., Gamache, R. R., Goldman. A., Brown, L. R., Toth, R. A., Picket. H. M.. Poynter. R., Flaud, J.-M., CamyPeyret, C., Barbe, A.. Husson, N., Rinsland, C. P. and Smith, M. A. H., The HITRAN datat base: 1986 cdition. Appl. Opt. 26. N19. 4058-4097, 1987.

Seiff, A., Post-liking models for the structure of the summer atmosphere of Mars. Adt. Spat' Re's. 2, 3. 1982.

Westley, F., Herron, J. T., Cretanovich, R. J., Hampson, R. F. 
and Mallard, W. G., NIST Chemical Kınetics Databaseversion 3.0. National Institute of Standards and Technology, 1991

\section{Appendix A}

\section{Nomlinearity correction}

The study of the i.r. speetrometer nonlincarity during the ground eallibrations resulted in the power law (Korablev et al. 1993):

$$
I_{I_{11}}=\frac{F}{F_{11}}\left[1-B\left(\begin{array}{c}
F \\
F_{11}
\end{array}\right)^{\prime \prime}\right]
$$

where $F$ is the solar flux, $I$ is the spectrometer rate, $F_{0}$ and $I_{0}$ correspond to the unattentated signal. and the numerical parameters are $B=0.042$ and $M=1.32$. For relative measurements the nonlinearity correction increases with the attenuation of the signal.

$$
T_{\text {corr }}=T^{1-B T^{\prime \prime}} \begin{gathered}
1-B \\
1-B
\end{gathered}
$$

$T$ and $T_{\text {corr }}$ are observed and corrected transmittances.

The individual nonlinearity of pixels is handled as a small modification to the general law (A2). The analysis of observational sequences with disturbed Sun-tracking is given by Chassefiere at al. (1992). We found sequence 2302235 to be the most beneficial in studying the individual nonlinearity. It demonstrates the best stability of solar flux in two stationary positions of the ficld of vicw at the solar disk: the first corresponds to regular pointing. and in the second the entire field of view is lighted (see Fig. A 1). During this sequence the sounded altitudes exceed $85 \mathrm{~km}$ and the galseous absorptions (except $\mathrm{CO}_{2}$ ) are negligible.

Comparing the shape of spectra at the two parts of the occultation curve (Fig. A1) we found that the increase of signal is dissimilar for different pixels. A spectral slope may oceur in irregular pointing conditions when the spectrometer slit is partially lighted. see Korablev et al. (1993a). However, the measured flux varies irregularly from pixel to pixel with an amplitude reaching $8 \%$. Therefore. the difference of spectra at the increased part of the occultation curve from the linear function (to account for the spectral slope mentioned above) reflects the effect of individual nonlinearity. We accepted the following approximation:

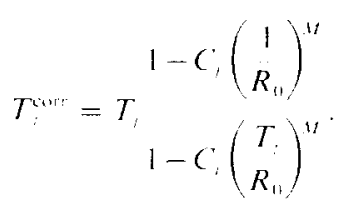

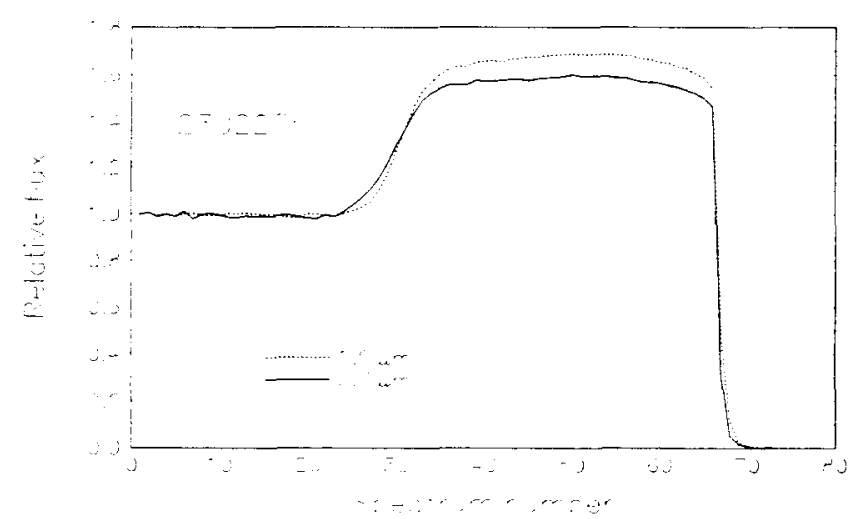

Fig. A1. An occultation sequence 2302235 demonst rating a steep increase of the measured flux
Here $R_{11}=1.695$ is the mean signal ratio of the two parts of the occultation curve and the corrections $C_{i}$ are chosen to fit the difference of spectra at the increased part from the straight line.

The uncertainty of the power law parameters defined in the calibrations is $\approx 3.5 \%$. The accuracy of the linear regression of spectra at the increased part of the occultation curve is $2 \%$. The error of factors $C$, is determined by these two values. The resulting uncertainty should be sealed according to attenuation for each measurement. according to the general nonlinearity law (A2). For slant optical depths relevant to the detection of formaldehyde the errors of nonlinearity correction are $0.5-1 \%$.

\section{Appendix B}

Periodical correction

We assume that a systematic error which needs correction results in the periodical linear transformation of the signal with the period of three pixels. The inverse transformation suppressing the error is therefore also periodical and proportional. It can be expressed as lollows:

$$
T_{i}^{(0) r}=T_{i}^{\prime \prime}\left(1+p_{k}\right) . \quad k=(j-1) \bmod 3+1,
$$

where $p_{k}$ are the correction parameters. $k=1,2.3$. If we fix the mean transmittance in the group of three consequent pixels, the inverse transformation is determined by two free parameters.

The contribution of periodical deformation can be evaluated with a discrete Fourier transform. In order to isolate a Fourier component related to the three-period structure we use the 15 point Fourier transform, and the 16 th pixel is withdrawn (it is partly shaded. see Section 2). All spectra corrected for the nonlincarity exhibit a distinet feature at the fifth Fourier component, as shown in Fig. Bl. We vary the parameter $p_{h}$ to find the inverse transformation suppressing this component (see Fig. B1).

It is interesting to trace the behavior of the derived corrections through the occultation (Fig. B2). They are negligible at the upper part of the occultation curve where the detector temperatures of measured and reference spectra are almost the same. The corrections $p_{k}$ derived independently for each measurement form a fairly smooth curve confirming that they are sensible.

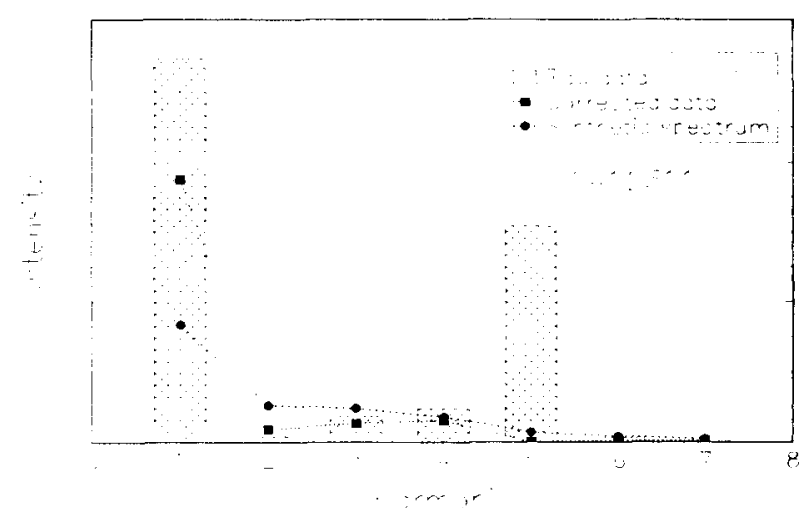

Fig. BI. The result of 15 -point discrete Fourier transform of a spectrum measured in sequence 2412311 before (bars) and after (squares) perindical correction procedure. The Fourier transform of the relevant synthetic spectrum is also shown (circles) 




Fig. B2. The parameters of periodical correction $p_{k}$ with error bars vs spectrum number in sequence 2412311
The regularity is lost beyond a certain point. This region corresponds to the lowest part of occultation with the slant optical thickness of 5 or more, where no absorptions can be identified due to the lower signal to noise ratio.

To find the error of the periodical correction we estimate how the suppression of the fifth harmonic in the Fourier transform affects the data. This harmonic calculated for the synthetic spectrum, corresponding to the detected mixing ratio of formaldehyde can be considered as the measure of the uncertainty. These uncertaintics are shown in Fig. B2 as crror bars. The same errors $(\approx 1 \%)$ are applied to the observed spectra.

Conclusively, the main sources of errors of the first-order spectra used for the identification of formaldehyde are the residual errors of periodical and nonlinearity correction $(\approx 1 \%$ and $\approx 0.5 \%$. respectively) and the random noise of the detector $(\approx 0.02 \%)$. The error bars of the mean spectra (Fig. 1) consist of the three forms of errors handled as independent. 